\title{
Retrospective, controlled observational case study of patients with central retinal vein occlusion and initially low visual acuity treated with an intravitreal dexamethasone implant
}

Sibylle Winterhalter ${ }^{*} \mathbb{D}$, Gerrit Alexander vom Brocke, Daniel Pilger, Annabelle Eckert, Juliane Schlomberg, Anne Rübsam, Matthias Karl Klamann, Enken Gundlach, Tina Dietrich-Ntoukas and Antonia Maria Joussen

\begin{abstract}
Background: Patients with initially low visual acuity were excluded from the therapy approval studies for retinal vein occlusion. But up to $28 \%$ of patients presenting with central retinal vein occlusion have a baseline BCVA of less than 34 ETDRS letters (0.1). The purpose of our study was to assess visual acuity and central retinal thickness in patients suffering from central retinal vein occlusion and low visual acuity $(<0.1)$ in comparison to patients with visual acuity $(\geq 0.1)$ treated with Dexamethasone implant $0.7 \mathrm{mg}$ for macular edema.

Methods: Retrospective, controlled observational case study of 30 eyes with macular edema secondary to central retinal vein occlusion, which were treated with a dexamethasone implantation. Visual acuity, central retinal thickness and intraocular pressure were measured monthly. Analyses were performed separately for eyes with visual acuity $<0.1$ and $\geq 0.1$.

Results: Two months post intervention, visual acuity improved only marginally from 0.05 to 0.07 ( 1 month; $p=0,065$ ) and to 0.08 ( 2 months; $p=0,2$ ) in patients with low visual acuity as compared to patients with visual acuity $\geq 0.1$ with an improvement from 0.33 to 0.47 ( 1 month; $p=0,005$ ) and to 0.49 ( 2 months; $p=0,003$ ). The central retinal thickness, however, was reduced in both groups, falling from 694 to $344 \mu \mathrm{m}$ ( 1 month; $p=0.003$,) to $361 \mu \mathrm{m}$ ( 2 months; $p=0,002)$ and to $415 \mu \mathrm{m}$ ( 3 months; $p=0,004)$ in the low visual acuity group and from 634 to $315 \mu \mathrm{m}$ ( 1 month; $p<0,001)$ and to $343 \mu \mathrm{m}$ ( 2 months; $p=0,001)$ in the visual acuity group $\geq 0.1$. Absence of visual acuity improvement was related to macular ischemia.
\end{abstract}

Conclusions: In patients with central retinal vein occlusion and initially low visual acuity, a dexamethasone implantation can lead to an important reduction of central retinal thickness but may be of limited use to increase visual acuity.

Keywords: Central retinal vein occlusion, Corticosteroids, Intravitreal dexamethasone implant, Macular edema, Visual acuity

* Correspondence: sibylle.winterhalter@charite.de

Department of Ophthalmology, Camus Virchow- Klinikum, Charité-University

Medicine Berlin, Augustenburger Platz 1, 13353 Berlin, Germany 


\section{Background}

Retinal vein occlusions (RVO) are the most common primary vascular diseases of the retina $[1,2]$. Branch retinal vein occlusions (BRVO) are more common than central retinal vein occlusions (CRVO) with a prevalence of 0.61.1 versus $0.1-0.4 \%$ in the general population [3, 4]. The spontaneous disease course is often poor especially in CRVO and hemi CRVO but also in BRVO. The majority of patients with retinal vein occlusion suffer from visual deterioration because of macular edema (ME). Quinlan [5] found in 1990 that only 13 to $17 \%$ of patients with CRVO had a significant visual acuity (VA) gain without treatment. The visual prognosis is thereby dependent on the initial VA and the degree of retinal ischemia [6]. An initial VA of $<20 / 200(<0.1)$ seems to be connected with a low VA prognosis. During the natural course of CRVO patients will suffer a medium VA impairment of 1 line VA chart [6].

The sham injection groups of the newer RVO therapy trials $[7,8]$ provide also data of the spontaneous disease course. Patients of the CRVO sham group lost in average 1 to 2 letters after 6 months of follow up during the GENEVA trial. In contrast to this the BRVO sham group gained 5 letters in average after 6 months [7, 8]. The GENEVA trial [7,8] excluded RVO patients with a BCVA of less than 34 ETDRS letters (0.1). But up to $28 \%$ of patients presenting with CRVO have a baseline BCVA of less than 34 ETDRS letters and more than $80 \%$ have a poor final VA if untreated [6].

The CRVO study group found in 1995 that grid pattern photocoagulation may reduce the leakage but without VA gain in CRVO patients with ME [9]. Since the Dexamethasone implant Ozurdex ${ }^{\oplus}$ (DEX implant) and the VEGF inhibitors Ranibizumab and Aflibercept were approved by the European Medicines Agency (EMA) in 2010, 2011 and 2013 for the treatment of ME secondary to RVO, the therapeutic options improved. However it is not clear if these new drugs have the capacity to improve the visual prognosis of patients with initial low VA $(<0.1)$. In this study CRVO patients with low VA have been treated with DEX implantation. The aim was to evaluate the outcome after intervention in this specific patient population.

\section{Methods}

In this retrospective, controlled, observational case study 30 eyes of 29 patients with ME secondary to CRVO were treated with a $700 \mu \mathrm{g}$ sustained delivery, bioerodable Dexamethasone intravitreal implant (DEX implant; Ozurdex ; Allergan, Inc., Irvine, CA) between November 2010 and July 2011. All patients gave their informed consent and all procedures were in concordance with the tenets of the Declaration of Helsinki. All patients were treated on label so that no ethics approval was needed for the retrospective chart review.

\section{Patients}

Patients with ME $>300 \mu \mathrm{m}$ secondary to CRVO were included into the study. Patients with known glaucoma were only included in case of well controlled intraocular pressure (IOP). Some patients received topical glaucoma medication after the CRVO occured because neuroprotection is suspected for e.g. carbonic anhydrase inhibiting eye drops $[10,11]$. Exclusion criteria were any ocular condition that was able to interfere with potential visual improvement and a pre-treatment with a DEX implantation at any time point. Analyses were performed separately for eyes with visual acuity $<0.1$ (15 eyes) and $\geq 0.1$ (15 eyes).

\section{Follow- up and treatment}

The baseline examinations included assessment of VA and IOP, complete ophthalmologic examination and central retinal thickness (CRT) measured by Spectral Domain Optical Coherence Tomography (SD-OCT) (Heidelberg Eye Explorer Version 6.011.0, Heidelberg Spectralis, Heidelberg Engineering, Heidelberg, Germany) before DEX implantation. Follow-up was conducted every 4 weeks over a time period of 6 months including VA, SD-OCT and complete ophthalmologic examination. A retreatment was performed mostly after 5 to 6 months if necessary so that this report refers only till month 5 after DEX implantation. Decimal visual acuity was converted into ETDRS letters with a VA conversion chart [12] to compare our results with the newer RVO therapy trials.

Side effects of the DEX implant were monitored by measuring the IOP every 4 weeks. Fluorescein angiography (FA) was performed in cases of missing contraindications (renal failure, reduced general condition, dye allergies) before DEX implantation or during the disease course to visualise macular and retinal perfusion. The FA procedure followed the multi-field protocol used by the Central Vein Occlusion Study Group, with standard 5- view angiography [13] using the HRA Spectralis (Heidelberg Eye Explorer Version 6.011.0, Heidelberg Engineering, Heidelberg, Germany) with a $55^{\circ}$ lens and the patients were asked to look in all directions to visualize the periphery. In cases of significant peripheral retinal ischemia patients were treated with panretinal photocoagulation to avoid neovascularisation and rubeotic secondary glaucoma. The peripheral retina was regarded as ischemic if ischemia amounted more than 10 disc diameters. Retreatment with DEX implantation or Ranibizumab was considered in cases of recurrent ME.

\section{Statistics}

Data were checked for normal distribution and results are expressed in mean \pm standard deviation (SD). Normallydistributed variables were compared with a matched paired sample $t$-test. Numeric variables that were not 
normally distributed were compared with the matched paired Wilcoxon signed rank test. The $\mathrm{Chi}^{2}$ - test was used to compare the gender differences between the groups. The results were regarded as statistically significant if $p$ was below 0.05. Statistics were performed with SPSS (Version 21.0.0.0, IBM, United States).

\section{Results}

\section{Patients}

\section{Low visual acuity group}

Six men and nine women with a baseline visual acuity $<0.1$ (15 eyes) were treated with a DEX implantation. The mean time after CRVO was $9( \pm 6.9)$ months in this group and the mean age of patients $72.5( \pm 9)$ years. Four patients $(26.7 \%)$ were pretreated with bevacizumab. Six patients $(40 \%)$ received topical glaucoma medication to protect the optic nerve head. One patient was diagnosed with chronic open angle glaucoma.

\section{Control group}

The control group consisted of seven men and eight women with a baseline visual acuity $\geq 0.1$ ( 15 eyes) and a mean time after CRVO of $9( \pm 7.3)$ months. The mean age of the control group amounted $72.3( \pm 9.9)$ years. Two patients (13.3\%) were pretreated with bevacizumab and one patient $(6.7 \%)$ was pretreated with bevacizumab and triamcinolone. Eight patients (53.3\%) received topical glaucoma medication at baseline. Two of these patients were diagnosed with chronic open angle glaucoma before treatment.

All DEX implantations were performed under standardized conditions (operating theatre, topical anaesthesia, sterile conditions, DEX implantation in tunnel technique in $3.5 \mathrm{~mm}$ distance of the limbus) without implantation related complications.

\section{Visual acuity}

\section{Low visual acuity group}

Throughout the follow up the distance VA of the low VA group improved compared to baseline (Table 1). VA improvement showed a weak effect but without statistical significance at any time point. The conversion into ETDRS letters is seen in Table 1, Figs. 1 and 2. The Additional file 1a-e shows in more detail the ETDRS letter change compared to baseline during month 1 to 5 after DEX implantation. A $\geq 15$ letter improvement was achieved in $40 \%$ of patients and $\mathrm{a} \geq 10$ letter improvement in $53.3 \%$ of patients at any time point.

\section{Control group}

In the control group, the distance VA assessment showed an improvement at month $1(p=0.005)$ and month $2(p=0.003)$ (Table 2). The conversion into ETDRS letters is shown in Table 2, Figs. 1 and 2 with
Table 1 VA and CRT in 15 CRVO patients with baseline VA $<0.1$

\begin{tabular}{llll}
\hline & Visual acuity & ETDRS letters $(V A)$ & $C R T(\mu \mathrm{m})$ \\
\hline Baseline $( \pm$ SD) & $0.05( \pm 0.03)$ & $20( \pm 12)$ & $694( \pm 301)$ \\
1 mo follow-up & $0.07( \pm 0.05)$ & $28( \pm 13)$ & $344( \pm 127)$ \\
$P$-value & 0.065 & 0.1 & $0.003^{*}$ \\
2 mo follow-up & $0.08( \pm 0.04)$ & $23( \pm 14)$ & $361( \pm 226)$ \\
$P$-value & 0.2 & 0.23 & $0.002^{*}$ \\
3 mo fyollow-up & $0.07( \pm 0.04)$ & $24( \pm 13)$ & $415( \pm 224)$ \\
$P$-value & 0.14 & 0.19 & $0.004^{*}$ \\
4 mo follow-up & $0.05( \pm 0.03)$ & $17( \pm 12)$ & $534( \pm 299)$ \\
$P$-value & 0.31 & 0.8 & 0.094 \\
5 mo follow-up & $0.06( \pm 0.04)$ & $20( \pm 12)$ & $665( \pm 340)$ \\
$P$-value & 0.93 & 0.82 & 0.14 \\
\hline
\end{tabular}

( ${ }^{*} p$-values with statistical significance)

the same effect from month $1(p=0.002)$ to month 2 $(p=0.013)$. The Additional file 2a-e shows in more detail the ETDRS letter change compared to baseline during month 1 to 5 after DEX implantation. A $\geq 15$ letter improvement was achieved in $46.7 \%$ of patients and $\mathrm{a} \geq 10$ letter improvement in $73.3 \%$ of patients at any time point.

\section{Central retinal thickness \\ Low visual acuity group}

The CRT assessed by SD-OCT was reduced compared to baseline with strong evidence for an effect during the first $(p=0.003)$, second $(p=0.002)$ and third month $(P=0.004)$ after DEX implantation (Table 1$)$.

\section{Control group}

In the control group the CRT was reduced compared to baseline again with a strong evidence for an effect from month $1(p<0.001)$ to month $2(p=0.001)$ (Table 2$)$.

\section{Fluorescein angiography and panretinal photocoagulation}

FA was performed in 14 eyes of the low VA group and 11 eyes of the control group before (18 eyes) or after DEX implantation (seven eyes). The remaining five patients did not receive a FA because of contraindications.

\section{Low visual acuity group}

FA showed macular ischemia in $64.3 \%$ of patients (Table 3, Fig. 3) shows a case report). Peripheral retinal ischemia was present in $84.6 \%$ of patients during FA likewise (Table 3, Fig. 3). One of the patients with macular ischemia did not have peripheral retinal ischemia and three other patients with perfused macular showed peripheral retinal ischemia. In total $57.1 \%$ of patients showed macular and peripheral retinal ischemia at the same time point. The periphery of one patient could not be evaluated 


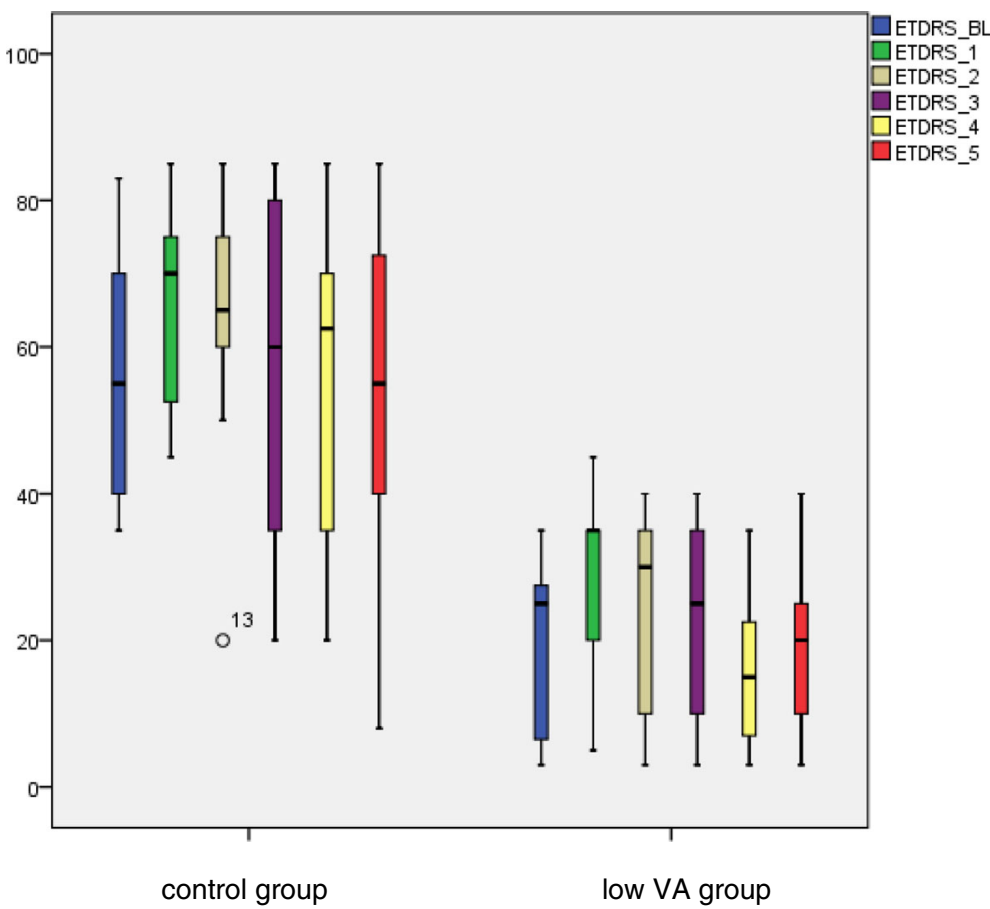

Fig. 1 Visual acuity course of the low visual acuity group in comparison to the control group after DEX implantation expressed in ETDRS letters with standard deviation

because of poor quality. All patients received panretinal photocoagulation (100\%).

\section{Control group}

Macular ischemia was present in $18.2 \%$ of patients (Table 3, $63.6 \%$ perfused, $18.2 \%$ not evaluable). Peripheral retinal ischemia could be detected in $36.4 \%$ of patients (Table 3, 36.4\% perfused, $27.3 \%$ not evaluable). Only one patient showed macular and peripheral retinal ischemia at the same time point. Eight patients were treated with panretinal photocoagulation (53.3\%).

\section{Intraocular pressure (IOP)}

\section{Low visual acuity group}

The median IOP was: $14.3 \pm 2.4 \mathrm{mmHg}$ (baseline); $18.5 \pm 2.6 \mathrm{mmHg}$ (month 1); $19.3 \pm 4 \mathrm{mmHg}$ (month 2 );
$17.1 \pm 1.6 \mathrm{mmHg}$ (month 3); $16.7 \pm 3 \mathrm{mmHg}$ (month 4 ) and $17.8 \pm 9.9 \mathrm{mmHg}$ (month 5). The IOP lowering medication had to be intensified in one patient with open angle glaucoma and in three further patients (26.7 \%).

\section{Control group}

In this group the median IOP was: $14.1 \pm 5.2 \mathrm{mmHg}$ (baseline); $18.9 \pm 8.2 \mathrm{mmHg}$ (month 1); $19.1 \pm 7.4 \mathrm{mmHg}$ (month 2); $16.8 \pm 3.5 \mathrm{mmHg}$ (month 3); $16.4 \pm 4 \mathrm{mmHg}$ (month 4) and $13 \pm 2.6 \mathrm{mmHg}$ (month 5). One patient with open angle glaucoma and 5 further patients needed an intensified IOP lowering medication (40\%).

The IOP raised to $\geq 25 \mathrm{mmHg}$ in $20 \%$ of patients and a $\geq 10 \mathrm{mmHg}$ increase of IOP from baseline at 60 days was seen in $13.3 \%$ of patients. Three patients suffered of IOP decompensation with $40 \mathrm{mmHg}$ in month 1

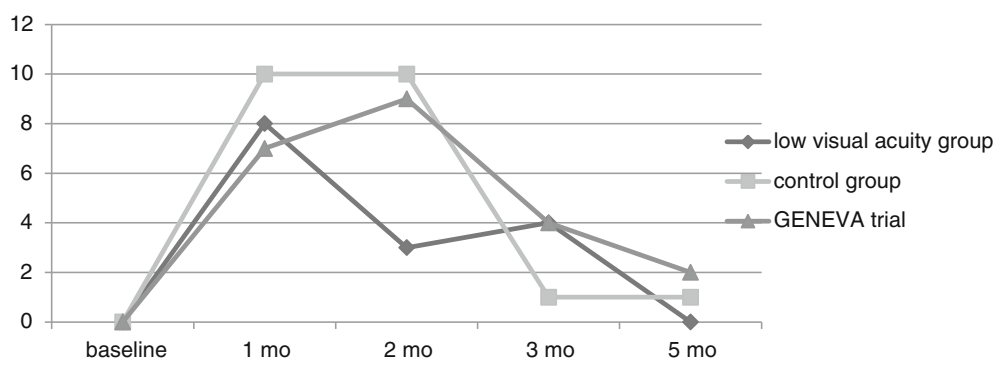

Fig. 2 Mean BCVA improvement in numbers of letters of the low visual acuity and control group in comparison to the GENEVA trial 
Table 2 VA ad CRT in 15 CRVO patients with baseline VA $\geq 0.1$

\begin{tabular}{llll}
\hline & Visual acuity & ETDRS letters $(V A)$ & $C R T(\mu \mathrm{m})$ \\
\hline Baseline $( \pm \mathrm{SD})$ & $0.33( \pm 0.27)$ & $55( \pm 17)$ & $634.38( \pm 130.40)$ \\
1 mo follow-up & $0.47( \pm 0.27)$ & $65( \pm 13)$ & $314.92( \pm 77.88)$ \\
P-value & $0.005^{*}$ & $0.002^{*}$ & $<0.001^{*}$ \\
2 mo follow-up & $0.49( \pm 0.29)$ & $65( \pm 25)$ & $343.27( \pm 73.89)$ \\
P-value & $0.003^{*}$ & $0.013^{*}$ & $0.001^{*}$ \\
3 mo follow-up & $0.44( \pm 0.37)$ & $56( \pm 25)$ & $483.17( \pm 255.16)$ \\
P-value & 0.24 & 1 & 0.18 \\
4 mo follow-up & $0.4( \pm 0.35)$ & $55( \pm 24)$ & $534.13( \pm 191.86)$ \\
$P$-value & 0.49 & 0.97 & 0.24 \\
5 mo follow-up & $0.39( \pm 0.39)$ & $54( \pm 26)$ & $490.5( \pm 76.15)$ \\
$P$-value & 0.13 & 0.77 & 0.23 \\
\hline
\end{tabular}

( ${ }^{*} p$-values with statistical significance)

(control group) and $38 \mathrm{mmHg}$ in month 2 (control group) after DEX implantation. One patient with an intraocular pressure of $35 \mathrm{mmHg}$ in month 5 showed rubeotic secondary glaucoma (low VA group) and was treated with retinal cryocoagulation during follow-up.

\section{Retreatment}

A retreatment was performed in 6 patients (40\%) of the low visual acuity group 4 to 9 months after DEX implantation with a medium retreatment time of 7 months.

Patients of the control group were retreated in $53.3 \%$ (8 patients) 5 to 8 months after DEX implantation with a medium retreatment time of 5.9 months.

\section{Discussion}

Our retrospective controlled study was performed to evaluate VA gain after DEX implantation in patients with initially low VA $<0.1$ because $\mathrm{CRVO}$ is often related to limited visual prognosis $[5,6,14]$ and the GENEVA trial $[7,8]$ excluded RVO patients with a BCVA of less than 34 ETDRS letters (0.1). Our results show that patients with initially low VA can profit of DEX implantation despite weak evidence for an effect: $40 \%$ of patients achieved $a \geq 15$ letter improvement and $53.3 \%$ of patients a $\geq 10$ letter improvement at any time point.

In contrast patients of the control group experienced VA amelioration with strong evidence for an effect from month $1(p=0.005)$ to month $2(p=0.003)$. This is in concordance to other studies $[15,16]$. The ETDRS letter gain of the control group was higher than in the low VA

Table 3 Macular and peripheral retinal ischemia of the low VA group in comparison to the control group

\begin{tabular}{lll}
\hline Ischemia visualized by FA & Low VA group & Control group \\
\hline Macular & $64.3 \%$ & $18.2 \%$ \\
Peripheral retinal & $84.6 \%$ & $36.4 \%$ \\
\hline
\end{tabular}

group with $46.7 \%$ of patients who gained $\geq 15$ letters and $73.3 \%$ of patients who improved with $\geq 10$ letters at any time point. During the GENEVA trial $[7,8]$ a $\geq 15$ letter improvement in BCVA from baseline was seen in up to $30 \%$ in the $0.7 \mathrm{mg}$ DEX group and at least $\mathrm{a} \geq 10$ letter improvement in up to $55 \%$ of eyes.

In opposition to our results Dinah [17] described in 19 CRVO patients with a baseline BCVA of less than 34 ETDRS letters that $70 \%$ of patients gained $\geq 15$ letters. In their study, up to a third of patients achieved a BCVA of 55 ETDRS letters after DEX implantation. In our study no patient of the low VA group achieved 55 ETDRS letters and only 1 patient achieved 45 ETDRS letters and another one 40 ETDRS letters.

The limited VA gain in our low VA group was related to macular ischemia which amounted up to $64.3 \%$.

In clinical experience macular ischemia is often not visible during FA in cases of diffuse edema. After ME resolution, the ischemia becomes apparent in FA. In contrast to the low VA group macular ischemia was present in $18.2 \%$ in the control group. A high correlation is known between low baseline VA and presence or development of ischemia [6]. Therefore, the benefit of patients with low VA and macular ischemia seems to be limited after DEX implantation. As Dinah et al. [17] did not evaluate FA, the retinal perfusion status of the cohort is not known.

Another recent study of Parodi examined DEX implants for macular edema secondary to ischemic retinal vein occlusions [18]. Fifteen patients with CRVO and macular and retinal ischemia were included in this study with a follow up of 12 months. The median ETDRS letter score of these patients was 10 at baseline. This is in contrast to our case series of 15 patients with low VA with a median ETDRS letter score of 20 at baseline. The CRVO patients of Parodis group gained 10 ETDRS letters after 1 month which is comparable with a letter gain of 8 ETDRS letters after 1 month in our patients. But in contrast to our patients, who worsened again at month 2, Parodis CRVO patients showed a steady letter gain till 37 ETDRS letters (0.1) at 12 months, which might be relevant for the patient but is limited too, through a mean number of 2.8 DEX implantations. All 15 patients of Parodis study were diagnosed with macular and retinal ischemia, which was the inclusion criterion. The inclusion criterion of our patients was a VA $<0.1$ to evaluate VA expectations in low VA patients because no informations are available of the approval studies for these patients. Parodis CRVO patients showed a CRT reduction from $749 \mu \mathrm{m}$ at baseline to $363 \mu \mathrm{m}$ at the 12 month examination. This is comparable to our patients with a CRT reduction from $694 \mu \mathrm{m}$ to the minimum CRT of $344 \mu \mathrm{m}$ on month 1 after DEX implantation. A reevaluation of the perfusion status of Parodis patients would 

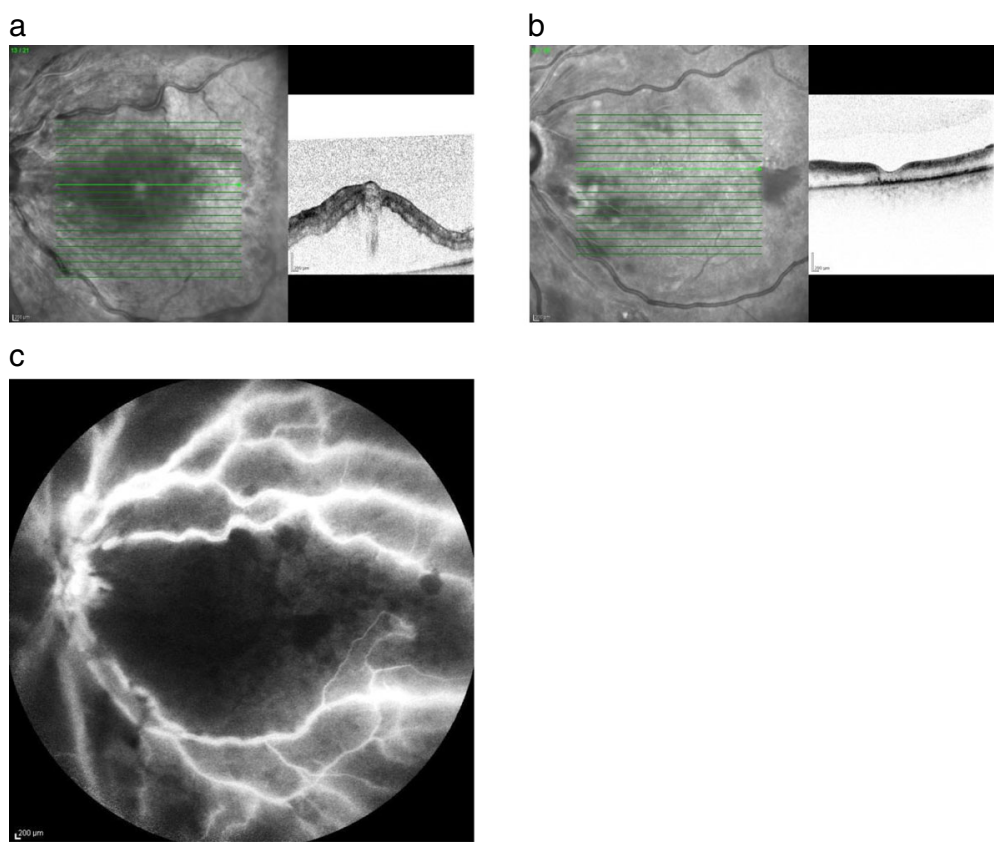

Fig. 3 a - c 81 year old lady, who suffered of CRVO with low VA on her left eye and was treated with a DEX implantation 2 months after the occlusion. a VA counted 3 ETDRS letters at baseline with a CRT of $924 \mu \mathrm{m}$ measured with the SD- OCT. b VA augmented to 35 ETDRS letters (0.1) 1 month after treatment with a reduced CRT of $214 \mu \mathrm{m}$. c VA gain was limited because the macula and retina were totally ischemic as seen in FA

have been interesting because of the steady letter gain till month 12. There are hints that aggressive blockade of VEGF with ranibizumab prevents the worsening of retinal non perfusion, promotes reperfusion and eliminates a positive feedback loop in patients with RVO [19].

Our results are in concordance with the study of Maggio [20] in which 21 eyes with CRVO, 20 eyes with BRVO and two eyes with hemiretinal vein occlusion (HRVO) were treated with a DEX implantation. They found that only the nonischemic CRVO subgroup had a statistically significant improvement in BCVA and CRT. The ischemic CRVO group did not improve significantly in BCVA in spite of significant improvements in CRT [20]. The CRT of our low VA group was reduced as well with strong evidence for an effect from month 1 to 3 $(p=0.003 ; p=0.002 ; p=0.004)$ despite a weak evidence for an effect concerning BCVA improvement. Other studies confirm that macular ischemia is a significant negative factor for VA improvement as described for intravitreal Bevacizumab for ME due to BRVO [21].

In our study the highest mean BCVA improvement in numbers of letters was similar to the GENEVA trial but our patients reached their highest letter gain earlier and dropped down earlier than the patients of the GENEVA trial. Effects of Dexamethasone implants are known in the literature as early as 7 days after injection regarding VA improvement and CRT reduction [22, 23]. Probably, an earlier treatment and retreatment might have been associated with a more favourable outcome.
Concerning the safety results a $\geq 10 \mathrm{mmHg}$ increase of IOP from baseline was seen in $13.3 \%$ of patients compared to $12.6 \%$ of patients at day 60 of the GENEVA trial $[7,8]$. In total the topical glaucoma- medication had to be intensified in $33.3 \%$ of patients of our study whereas $25.5 \%$ of patients began an IOP lowering medication during the masked phase of the GENEVA trial $[7,8]$.

A combination therapy of vascular endothelial growth factor (VEGF) inhibitors and DEX implants might have been associated with better VA results because the medium CRT was higher than $300 \mu \mathrm{m}$ after DEX implantation in both groups of our study at any time point. Regarding the literature a combination therapy of vascular endothelial growth factor (VEGF) inhibitors and DEX implants improved significantly the VA outcome in BRVO patients but not in CRVO patients [24]. However, the approval studies for VEGF inhibitors [25-27] to treat ME due to RVO showed a higher ETDRS letter gain than the GENEVA trial $[7,8]$. A combination therapy may lead to higher VA gain despite fewer necessary injections than with VEGF inhibitor therapy alone [28-31] and may be therefore cost-effective [32]. Despite this a combination therapy would not resolve the macular and retinal ischemia which was associated to limited final VA in our study. Otherwise CRVO patients of the CRYSTAL study with macular ischemia showed a VA improvement of 11.6 ETDRS letters under 3 primary Ranibizumab injections and following PRN treatment after 12 months which was comparable to patients 
without macular ischemia (12.1 ETDRS letters) [33]. But the inclusion criterion of the CRYSTAL study was a baseline BCVA of 19 to 73 ETDRS letters (0.05-0.5) whereas the inclusion criterion for our low VA group was a VA $<34$ ETDRS letters $(<0.1)$. So a more severe macular ischemia is suspected in our patients than that of the patients of the CRYSTAL study or Ranibizumab might be more effective in CRVO patients with macular ischemia than DEX implants.

Our study has a number of limitations. First, this was a retrospective study, and therefore we do not have full control over confounding factors. Also, our results have to be interpreted with caution due to the small sample size which resulted in low statistical power. Hence, interpretation of our findings should consider observed trends and not entirely rely on statistical significance. These trends are largely similar to the trends bowered in larger studies such as the GENEVA trial (Fig. 2) which makes a chance finding unlikely. A further limitation results in the mean duration of CRVO which was 9 months in the low VA group and could have resulted in a lower VA gain. But it has to be said that the mean duration of CRVO was 9 months in the control group as well which showed a similar ETDRS letter gain than the patients of the GENEVA trial. This study was performed shortly after DEX implants were approved in Europe for the treatment of RVO, so the duration of CRVO was longer in some patients and some patients were pretreated with bevacizumab. But the baseline characteristics of the control group were similar to the low VA group so that our results seem to be realistic. Our results showed as well, that some patients should have been retreated earlier as described before [16], which was a learning effect.

\section{Conclusion}

VA prognosis is limited after DEX implantation in CRVO patients with initial low VA because of macular ischemia. Despite the limited VA prognosis we would recommend to treat patients due to the fact that these patients showed a slight benefit in our study. However, patients should be informed about the limited VA prognosis to avoid unrealizable expectations. The results may help to prevent risks and burden for the patients and costs for the health care system in patients with CRVO and low VA.

\section{Additional files}

Additional file 1: a-c) waterfall plots of the ETDRS letter change 1 to 5 months after DEX implantation compared to baseline of the low VA group. (DOCX $48 \mathrm{~kb}$ )

Additional file 2: $\mathrm{a}-\mathrm{c}$ ) waterfall plots of the ETDRS letter change 1 to 5 months after DEX implantation compared to baseline of the control group. (DOCX $48 \mathrm{~kb}$ )

\section{Abbreviations}

BCVA: Best corrected visual acuity; BRVO: Branch retinal vein occlusion; CRT: Central retinal thickness; CRVO: Central retinal vein occlusion; DEX implant: Dexamethasone implant; EMA: European medicines agency; ETDRS: Early treatment diabetic retinopathy study; FA: Fluorescein angiography; IOP: Intraocular pressure; ME: Macular edema; RVO: Retinal vein occlusio; SD: Standard deviation; SD-OCT: Spectral domain optical coherence tomography; VA: Visual acuity; VEGF: Vascular endothelial growth factor

\section{Acknowledgements}

We want to thank Mr. Dirk Scharf for his excellent technical assistance.

\section{Funding}

The whole study was performed without any funding.

Availability of data and materials

Data supporting our findings are contained in the manuscript.

\section{Authors'contribution}

SW designed the study, performed the patients examinations and the analysis and wrote and drafted the manuscript. GAvB performed the statistical analysis. DP is MSc in Epidemiology and advised and revised the statistical analysis and the manuscript. AE participated in the patient analysis. JSch revised the manuscript critically. AR revised the manuscript critically. MKK revised the manuscript critically. EG revised the manuscript critically. TD revised the manuscript critically. AMJ revised the manuscript critically. All authors read and approved the final manuscript.

\section{Competing interests}

The authors declare that they have no competing interests.

\section{Consent for publication}

Not applicable.

\section{Ethics approval and consent to participate}

All patients gave their informed consent and all procedures were in concordance with the tenets of the Declaration of Helsinki. All patients were treated on label so that no ethics approval was needed for the retrospective chart review.

Received: 20 February 2016 Accepted: 13 October 2016

Published online: 27 October 2016

References

1. Feltgen N, Pielen A, Hansen L, Bertram B, Agostini H, Jaissle GB, Hoerauf H, Stahl A. Intravitreal drug therapy for retinal vein occlusion- pathophysiological mechanisms and routinely used drugs. Klin Monatsbl Augenheilkd. 2010;227:681-93.

2. Hansen L, Feltgen N. Central retinal vein occlusion, Branch retinal vein occlusion. In: Retinal vascular diseases, Joussen AM editor. Berlin: 1st edn Springer Verlag; 2012. p. 189-224.

3. Mitchell P, Smith W, Chang A. Prevalence and associations of retinal vein occlusion in Australia. The Blue Mountains Eye Study. Arch Ophthalmol. 1996;114:1243-7.

4. Klein R, Klein BE, Moss SE, Meuer SM. The epidemiology of retinal vein occlusion: the Beaver Dam Eye Study. Trans Am Ophthalmol Soc. 2000;98:133-41.

5. Quinlan PM, Elman MJ, Bhatt AK, Mardesich P, Enger C. The natural course of central retinal vein occlusion. Am J Ophthalmol. 1990;110:118-23.

6. The Central Vein Occlusion Study Group. Natural history and clinical management of central retinal vein occlusion. Arch Ophthalmol. 1997;115:486-91.

7. Haller JA, Bandello F, Belfort R, Blumenkranz MS, Gillies M, Heier J, Loewenstein A, Yoon YH, Jarques ML, Jiao J, Li XY, Whitcup SM, OZURDEX GENEVA Study Group. Randomized, sham- controlled trial of dexamethasone intravitreal implant in patients with macular edema due to retinal vein occlusion. Ophthalmology. 2010;117:1134-46.

8. Haller JA, Bandello F, Belfort R, Blumenkranz MS, Gillies M, Heier J, Loewenstein A, Hee Yoon Y, Jiao J, Whitcup SM for the Ozurdex GENEVA Study Group. Dexamethasone Intravitreal Implant in Patients with Macular Edema Related to Branch or Central Retinal vein Occlusion. Ophthalmology. 2011;118:2453-60. 
9. The Central Vein Occlusion Study Group. Evaluation of grid pattern photocoagulation for macular edema in central vein occlusion. The Central Vein Occlusion Study Group M report. Ophthalmology. 1995;102:1425-33.

10. Sugrue MF. The preclinical pharmacology of dorzolamide hydrochloride, a topical carbonic anhydrase inhibitor. J Ocul Pharmacol Ther. 1996;12(3):363-76.

11. Park HY, Lee NY, Kim JH, Park CK. Intraocular pressure lowering, change of antiapoptotic molecule expression, and neuroretinal changes by dorzolamide $2 \% /$ timolol $0.5 \%$ combination in a chronic ocular hypertension rat model. J Ocul Pharmacol Ther. 2008;24(6):563-71.

12. Rosser DA, Laidlaw, Murdoch IE. The development of a "reduced logMAR" visual acuity chart for use in routine clinical practice. Br J Ophthalmol. 2001;85:432-6.

13. The Central Vein Occlusion Study Group. A randomized clinical trial of early panretinal photocoagulation for ischemic central vein occlusion. The CVOS Group N Report. Ophthalmology. 1995;102:1434-44.

14. Mclntosh RL, Rogers SL, Lim L, Cheung N, Wang JJ, Mitchell P, Kowalski JW, Nguyen HP, Wong TY. Natural history of central retinal vein occlusion: an evidence- based systematic review. Ophthalmology. 2010;117:1113-23.

15. Querques G, Lattanzio R, Querques L, Triolo G, Cascavilla M, Cavallero E, del Turco C, Casalino G, Bandello F. Impact of intravitreal dexamethasone implant (Ozurdex) on macular morphology and function. Retina. 2014;34:330-41

16. Winterhalter S, vom Brocke GA, Klamann MK, Müller B, Joussen AM. Monthly microperimetry (MP1) measurement of macular sensitivity after dexamethasone implantation (Ozurdex) in retinal vein occlusions. Graefes Arch Clin Exp Ophthalmol. 2015;253(11):1873-82.

17. Dinah C, Nenova K, Pushpoth S, El-Ghrably I, Varma D, Talks SJ. Intravitreal dexamethasone for central retinal vein occlusion with low visual acuity: a retrospective study. Br J Ophthalmol. 2013;97:796-7.

18. Parodi MB, lacono P, Petruzzi G, Parravano M, Varano M, Bandello F. Dexamethasone implant for macular edema secondary to ischemic retinal vein occlusions. Retina. 2015:35:1387-92.

19. Campochiaro PA, Bhisitkul RB, Shapiro H, Rubio RG. Vascular Endothelial Growth Factor Promotes Progressive Retinal Nonperfusion in Patients with Retinal Vein Occlusion. Ophthalmology. 2013;120:795-802.

20. Maggio E, Plito A, Guerriero M, Pertile G. Intravitreal Dexamethasone Inplant for macular Edema Secondary to Retinal Vein Occlusion: 12- month Follow- Up and prognostic Factors. Ophthalmologica. 2014;232:207-15.

21. Chung EJ, Hong YT, Lee SC, Kwon OW, Koh HJ. Prognostic factors for visual outcome after intravitreal bevacizumab for macular edema due to branch retinal vein occlusion. Graefes Arch Clin Exp Ophthalmol. 2008;246:1241-7.

22. Kuppermann BD, Haller JA, Bandello F, Loewenstein A, Jiao J, Li XY, Whitcup SM. Onset and duration of visual acuity improvement after dexamethasone intravitreal implant in eyes with macular edema due to retinal vein occlusion. Retina. 2014;34:1743-9.

23. Veritti D, Macor S, Lanzetta P. Early Effects of Dexamethasone Implant on Macular Morphology and Visual Function in Patients with Macular Edema Secondary to Retinal Vein Occlusion. Ophthalmologica. 2014;232:144-8.

24. Iu LP, Zhao P, Yeung IY, Fung NS, Lee JW, Wong RL, Chong V, Wong IY. Sequential therapy with ranibizumab and dexamethsone intravitreal implant is better than dexamethasone monotherapy for macular oedema due to retinal vein occlusion. $\mathrm{Br} J$ Ophthalmol. 2015;99:210-4.

25. Brown DM, Heier JS, Clark WL, Boyer DS, Vitti R, Berliner AJ, Zeitz O, Sandbrink R, Zhu X, Haller JA. Intravitreal aflibercept injection for macular edema secondary to central retinal vein occlusion: 1 - year results from the phase 3 COPERNICUS study. Am J Ophthalmol. 2013;155:429-37.e7.

26. Holz FG, Roider J, Ogura Y, Korobelnik JF, Simader C, Groetzbach G, Vitti R, Berliner AJ, Hiemeyer F, Beckmann K, Zeitz O, Sandbrink R. VEGF Trap- Eye for macular oedema secondary to central retinal vein occlusion. 6-month results of the phase III GALILEO study. Br J Ophthalmol. 2013;97:278-84.

27. Brown DM, Campchiaro PA, Singh RP, Li Z, Gray S, Saroj N, Rundlle AC, Rubio RG, Murahashi WY. CRUISE investigators. Ranibizumab for macular edema following central retinal vein occlusion: six- month primary end point results of a phase III study. Ophthalmology. 2010;117:1124-33.

28. Maturi RK, Chen V, Raghinaru D, Bleau L, Stewart MW. A 6-month, subjectmasked, randomized controlled study to assess efficacy of dexamethasone as an adjunct to bevacizumab compared with bevacizumab alone in the treatment of patients with macular edema due to central or branch retina vein occlusion. Clin Ophthalmol. 2014;8:1057-64.
29. Nghiem-Buffet S, Fajnkuchen F, Buffet M, Ayrault S, Le Gloahec-Lorcy A, Grenet T, Delahye- Mazza C, Quentel G, Cohen SY. Intravitreal Ranibizumab and/or Dexamethasone Implant for Macular Edema Secondary to Retinal Vein Occlusion. Ophthalmologica. 2014;232:216-22.

30. Ozkok A, Saleh OA, Sigford DK, Heroman JW. THE OMAR STUDY: Comparison of Ozurdex and Triamcinolone Acetonide for Refractory Cystoid Macular Edema in Retinal Vein Occlusion. Retina. 2015;35:1393-400.

31. Singer MA, Bell DJ, Woods P, Pollard J, Boord T, Herro A, Porbandarwalla S. Effect of combination therapy with bevacizumab and dexamethsone intravitreal implant in patients with retinal vein occlusion. Retina. 2012:32(7):1289-94.

32. Lozana López V, Serrano García M, Mantolán Sarmiento C, Pareja Ríos A Losada Castillo MJ, Cordovés Dorta L, Quijada Fumero E, Virgós Aller T, Bullejos Molina M. A cost- effectiveness study of dexamethsaone implants in macular edema. Arch Soc Esp Oftalmol. 2015;90:14-21.

33. Larsen M, Waldstein SM, Waldstein SM, Boscia F, Gerding H, Monés J, Tadayoni R, Priglinger S, Wenzel A, Barnes E, Pilz S, Stubbings W, Pearce I, CRYSTAL Study Group. Individualized Ranibizumab Regimen Driven by Stabilization Criteria for Central Retinal Vein Occlusion: Twelve-Month Results of the CRYSTAL Study. Opthalmology. 2016;123(5):1101-11.

\section{Submit your next manuscript to BioMed Central and we will help you at every step:}

- We accept pre-submission inquiries

- Our selector tool helps you to find the most relevant journal

- We provide round the clock customer support

- Convenient online submission

- Thorough peer review

- Inclusion in PubMed and all major indexing services

- Maximum visibility for your research

Submit your manuscript at www.biomedcentral.com/submit
) Biomed Central 